\title{
İki serbestlik dereceli bir robot kolun konum kontrolü için PID kontrol parametrelerinin arı algoritması (AA) kullanılarak belirlenmesi
}

\author{
Erdem KARAKOYUN ${ }^{1, *}$, Abdullah ÇAKAN ${ }^{1}$, Mete KALYONCU ${ }^{1}$ \\ ${ }^{1}$ Konya Teknik Üniversitesi Müh.-Mim. Fak. Makina Müh. Böl., Selçuklu, KONYA.
}

Geliş Tarihi (Received Date): 22.02.2021

Kabul Tarihi (Accepted Date): 05.08.2021

$\ddot{\mathbf{O} z}$

Bu çalışmada, bir robot kolun konum kontrolü için PID kontrol parametreleri Genetik algoritma (GA) ve Art Algoritması (AA) kullantlarak belirlenmiştir. Robot kolun dinamik ve kinematik denklemleri elde edilerek matematiksel modeli oluşturulmuştur. $G A$ ve AA ile PID kontrol parametreleri belirlenirken minimum konum hatasi sağlanabilmesi amaçlanmıştır. Optimizasyon ve benzetim çalışmaları Matlab/Simulink ortamında yapılmıştır ve elde edilen sonuçlardan; Her iki algoritmasının da PID kontrol parametrelerinin belirlenmesinde başarll olduğu ve robot kolun istenilen referans konumlara gelebildiği görülmüştür. Ancak, Arı Algoritması ile elde edilen sonuçlarda daha az maksimum aşmalar gözlenmiştir.

Anahtar kelimeler: Arı algoritması, PID kontrol, optimizasyon, robotik, manipülatör.

\section{Determination of PID control parameters for position control of a 2-dof robot arm using the bees algorithm (BA)}

\begin{abstract}
In this study, PID control parameters for position control of a robot arm were determined using Genetic Algorithm (GA) and The Bees Algorithm (BA). The mathematical model of the robot arm is created by obtaining the dynamic and kinematic equations. While determining PID control parameters with GA and BA, it is aimed to provide minimum position error. Optimization and simulation studies is realized in Matlab/Simulink environment and the results are shown that both algorithms are

\footnotetext{
*Erdem KARAKOYUN, karakoyun.erdem@ @otmail.com, https://orcid.org/0000-0002-5419-7430 Abdullah ÇAKAN, acakan@ktun.edu.tr, https://orcid.org/0000-0003-3923-4069 Mete KALYONCU, mkalyoncu@ktun.edu.tr, https://orcid.org/0000-0002-2214-7631
} 
successful in determining the PID control parameters and the robot arm can reach the desired reference positions. However, less maximum overshoots are observed in the results obtained with The Bees Algorithm.

Keywords: The bees algorithm, PID control, optimization, robotics, manipulator.

\section{Giriş}

İnsanlar kendilerine zor gelen işleri kolaylaştırmak veya yapamayacağı işleri yaptırmak için çeşitli yollar aramışlardır. Başlarda sömürebilecekleri insan gücünü kullanmışlardır. İnsan gücünün verimsiz ya da yetersiz kaldığı işlerle bahşetmek için ise ihtiyaçlar doğrultusunda çeşitli araç ve gereçler geliştirmeyi akıl etmişlerdir. Robotlar bu ihtiyaçları giderebilecek en gelişmiş araçlardır. İlk defa Çekoslovak yazar Karel Capek Rossum tarafından kullanılan "Robot" kelimesi, Çekoslovak'ça' da "zorla çalıştırılan işçi anlamına gelmektedir. Robot kelimesinin kökenine indiğimizde bile temel amacının insana hizmet etmek olduğunu anlamaktayız [1].

Günümüzde robotlar, endüstride yaygın bir şekilde kullanılmaktadır ve akademik çalışmalarda çokça yer bulmaktadır. Literatürde farklı amaçlara yönelik çok çeşitli konfigürasyonlarda robot çalışmalarına rastlanmaktadır. İki ve üç serbestlik dereceli robot çalışmaları literatürde daha fazla karşımıza çıkmasının yanı sıra dört ve daha fazla serbestlik derecesine sahip robot çalışmaları da çokça bulunmaktadır [2-6]. Robotlarda serbestlik derecesi arttıkça dinamik ve kinematik yapıları zorlaşmaktadır bu yüzden bilgisayar destekli çözümleme ve modelleme araçları robot çalışmalarında kolaylık sağlamaktadır [7]. İki serbestlik dereceli robot kol modeli robot çalışmalarında temel oluşturmaktadır. Kendi başına kullanılabileceği gibi bir robot sisteminin de bir parçası olabilmektedir. $\mathrm{Bu}$ yüzden iki serbestlik dereceli sistem üzerinde başarılı sonuçlar alabilmek daha sonra karmaşık robot sistemlerinin geliştirilmesi üzerine yapılacak çalışmalarda başarı oranını artıracaktır [8].

Robotların doğru modellenmesi doğru kontrol çalışması yapabilmek adına çok önemlidir. Konum kontrolü, yörünge takibi gibi çalışmalar uzun yıllardır araştırmacıların robotik çalışmalarına konu olmuştur. Bu yüzden PID, LQR, Kayan Kipli Kontrol gibi çeşitli kontrolcüler geliştirilerek, farklı amaçlar için uygulanmıştır [913]. Kontrolcü tasarımında genellikle geleneksel yöntemler kullanılmaktadır, bu yöntemlerle elde edilen kontrol parametreleri hangi parametrenin ne derece etkili oldukları konusunda bakış açısı sağlamaktadır [14]. Tasarlanan kontrolcüler, bu bakış açısı ile parametreler üzerinde deneme yanılma yoluyla değişiklikler yapılarak geliştirilebilmektedir. Optimizasyon algoritmaları ile kontrol problemine yönelik amaç fonksiyonları, sinırlar ve kisitlar belirlenerek, kontrol parametrelerinin daha kolay bir şekilde elde edilmesine yardımcı olabildiği gibi daha iyi sonuçlara ulaşılmasını da sağlayabilmektedir. Literatürde yaygın olarak rastlanan Genetik Algoritma (GA) ve Parçacık Sürü Algoritması (PSO) gibi algoritmaların yanı sıra Yapay Arı Algoritması $(\mathrm{ABC})$, Yanıt Yüzey Yöntemi (YYY) gibi algoritma ve yöntemlerin de kontrolcü parametrelerinin belirlenmesinde kullanıldığına rastlanmıştır [15-20].

$\mathrm{Bu}$ çalışmada, bir robot kolun konum kontrolü için PID kontrol parametreleri Genetik Algoritma (GA) ve Arı Algoritması (AA) kullanılarak belirlenmiştir. Robot kolun matematiksel modeli oluşturulmak üzere dinamik ve kinematik denklemleri elde 
edilmiştir. GA ve AA ile PID kontrol parametreleri belirlenirken dinamik modelin basamak girişine karşılık zaman cevapları elde edilerek, amaç fonksiyonu olarak yükselme zamanı, tepe zamanı, yerleşme zamanı, maksimum aşma ve kalıcı durum hatası toplanarak oluşturulmuştur ve bu sayede konum hatasının en aza indirgenmesi amaçlanmıştır. Optimizasyon ve benzetim çalışmaları yapıldıktan sonra elde edilen sonuçlara göre Arı Algoritmasının PID kontrol parametrelerinin belirlenmesinde başarılı olduğu ve robot kolun referans konumlara daha az aşmalar ile gelebildiği görülmüştür.

\section{Sistemin matematiksel modeli ve kontrolü}

$\mathrm{Bu}$ bölümde iki serbestlik dereceli robot kolun matematiksel modeli elde edilerek kontrol sistemi tasarlanmıştır. Matematiksel modeli elde edilen iki serbestlik dereceli robot kolun şematik gösterimi Şekil 1'de, robot kola ait parametreler ise Tablo 1'de verilmiştir.

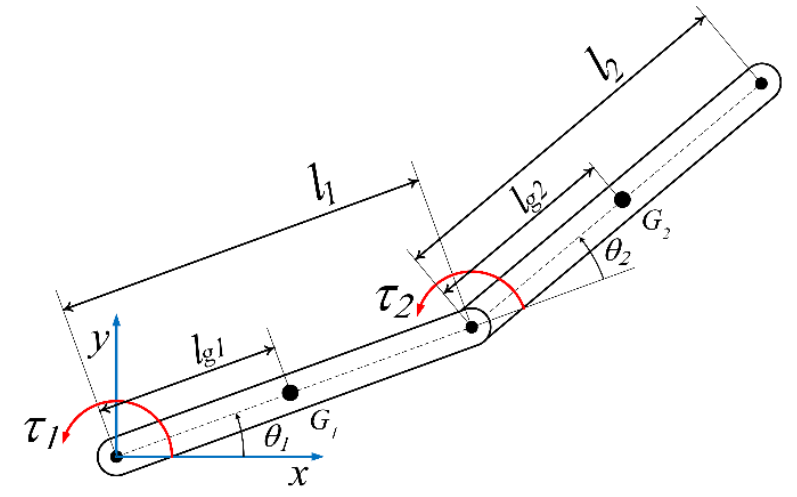

Şekil 1. İki serbestlik dereceli robot.

Tablo 1. İki serbestlik dereceli robot tasarım parametreleri.

\begin{tabular}{|c|c|c|}
\hline$l_{1}$ & 1.uzuv uzunluğu & $1 \mathrm{~m}$ \\
\hline$l_{2}$ & 2. uzuv uzunluğu & $1 \mathrm{~m}$ \\
\hline$l_{g 1}$ & 1.uzuv ağırlık merkezi & $0.5 \mathrm{~m}$ \\
\hline$l_{g 2}$ & 2. uzuv ağırlık merkezi & $0.5 \mathrm{~m}$ \\
\hline$m_{1}$ & 1. uzuv ağırlığ & $1 \mathrm{~kg}$ \\
\hline$m_{2}$ & 2. uzuv ağırlığ1 & $1 \mathrm{~kg}$ \\
\hline$\theta_{1}$ & 1. uzuv açısal konumu & Derece \\
\hline$\theta_{2}$ & 2. uzuv açısal konumu & Derece \\
\hline$\tau_{1}$ & 1. uzuv torku & $\mathrm{Nm}$ \\
\hline$\tau_{2}$ & 2. uzuv torku & $\mathrm{Nm}$ \\
\hline$I_{1}$ & 1.uzuv ağırlık merkezine göre kütle atalet momenti & $\mathrm{kgm}^{2}$ \\
\hline$I_{2}$ & 2.uzuv ağırlık merkezine göre kütle atalet momenti & $\mathrm{kgm}^{2}$ \\
\hline
\end{tabular}

\subsection{Dinamik model}

Robot kol sisteminin hareket denklemi elde edilirken Lagrange mekaniğinden yararlanılmıştır. Lagrange mekaniğinde ilk olarak Lagrangian Fonksiyonu elde edilir, 
Lagrangian Fonksiyonu sistemin kinetik ve potansiyel enerji denklemlerinin fark1 alınarak bulunur. Lagrangian Fonksiyonunun genel ifadesi Eşitlik (1)'de verilmiştir.

$L=K E-P E$

Sistemin Kinetik enerji, potansiyel enerji ve bunların farkından elde edilen Lagrangian Fonksiyonu eşitlikleri sırasıyla Eşitlik (2), (3) ve (4)'de verilmiştir. Lagrangian Fonksiyonu vasıtasıyla sistemin hareket denklemleri türetilmiştir.

$$
\begin{aligned}
& K E=\frac{1}{2} m_{1} l_{g_{1}}^{2} \dot{\theta}_{1}^{2}+\frac{1}{2} m_{2}\left(l_{1}^{2} \dot{\theta}_{1}^{2}+2 l_{1} l_{g_{2}} \dot{\theta}_{1}\left(\dot{\theta}_{1}+\dot{\theta}_{2}\right) \cos \theta_{2}+l_{g_{2}}^{2}\left(\dot{\theta}_{1}+\dot{\theta}_{2}\right)^{2}\right)+\frac{1}{2} I_{1} \dot{\theta}_{1}{ }^{2}+\frac{1}{2} I_{2}\left(\dot{\theta}_{1}+\dot{\theta}_{2}\right)^{2} \\
& P E=m_{1} g l_{g_{1}} \sin \theta_{1}+m_{2} g\left(l_{1} \sin \theta_{1}+\left(l_{g_{2}} \sin \left(\theta_{1}+\theta_{2}\right)\right)\right. \\
& L=\frac{1}{2} m_{1} l_{g_{1}}^{2} \dot{\theta}_{1}{ }^{2}+\frac{1}{2} m_{2}\left(l_{1}^{2} \dot{\theta}_{1}{ }^{2}+2 l_{1} l_{g_{2}} \dot{\theta}_{1}\left(\dot{\theta}_{1}+\dot{\theta}_{2}\right) \cos \theta_{2}+l_{g_{2}}^{2}\left(\dot{\theta}_{1}+\dot{\theta}_{2}\right)^{2}\right)+\frac{1}{2} I_{1} \dot{\theta}_{1}+ \\
& \quad \frac{1}{2} I_{2}\left(\dot{\theta}_{1}+\dot{\theta}_{2}\right)^{2}-\left(m_{1} g l_{g_{1}} \sin \theta_{1}+m_{2} g\left(l_{1} \sin \theta_{1}+\left(l_{g_{2}} \sin \left(\theta_{1}+\theta_{2}\right)\right)\right)\right.
\end{aligned}
$$

Bir robot kolun genel tork denklemi Eşitlik (5)'deki gibidir. Lagrangian Fonksiyonu bu denkleme göre genelleştirilerek sistemin hareket denklemleri türetilmiştir. Türetilen hareket denklemleri matris formunda Eşitlik (6)'da verilmiştir.

$$
\begin{aligned}
& \tau=M(q) \ddot{q}+C(\dot{q}, q)+G(q) \\
& {\left[\begin{array}{l}
\tau_{1} \\
\tau_{2}
\end{array}\right]=\left[\begin{array}{cc}
m_{1} l_{g_{1}}^{2}+m_{2} l_{1}^{2}+I_{1} & m_{2} l_{1} l_{g_{2}} \cos \left(\theta_{1}-\theta_{2}\right) \\
m_{2} l_{1} l_{g_{2}} \cos \left(\theta_{1}-\theta_{2}\right) & m_{2} l_{g_{2}}^{2}+I_{2}
\end{array}\right]\left[\begin{array}{l}
\ddot{\theta}_{1} \\
\ddot{\theta}_{2}
\end{array}\right]-} \\
&\left(m_{2} l_{1} l_{g_{2}} g \sin \left(\theta_{1}-\theta_{2}\right)\left[\begin{array}{c}
\dot{\theta}_{1} \\
\dot{\theta}_{2}
\end{array}\right]+\left[\begin{array}{c}
\left(m_{1} l_{g_{1}}+m_{2} l_{1}\right) g \cos \theta_{1} \\
m_{2} l_{g_{2}} g \cos \theta_{2}
\end{array}\right]\right.
\end{aligned}
$$

Robot kolun hareket denklemleri lineerleştirilerek durum uzay modeli elde edilir. Elde edilen durum uzay modeline ait A, B, C ve D matrisleri Eşitlik 7'de verilmiştir. Durum uzay modeli PID kontrolcü tasarımında ve basamak girişi uygulanarak GA ve AA ile PID parametrelerinin belirlenmesinde kullanılmıştır [21].

$$
\begin{array}{rlrl}
A & =\left[\begin{array}{ccc}
0 & 0 & 10 \\
0 & 0 & 01 \\
-0,4568 & -0,6196 & 0 \\
0,2485 & -6,6174 & 0
\end{array}\right] & B=\left[\begin{array}{cc}
0 & 0 \\
0 & 0 \\
0,7870 & -0,0426 \\
0,0426 & 0,1349
\end{array}\right] \\
C=\left[\begin{array}{lll}
1 & 00 & 0 \\
0 & 10 & 0
\end{array}\right] & D=\left[\begin{array}{ll}
0 & 0 \\
0 & 0
\end{array}\right]
\end{array}
$$

\subsection{Kinematik model}

Dinamik model mafsallardaki torklar ve açısal konumları arasındaki eşitliklerden oluşmaktadır, ancak kinematik model açısal konumlar ile robotun uç noktasının arasındaki eşitliklerden oluşur. Kinematik model; ters ve düz kinematik olmak üzere iki durumdan oluşur. Düz kinematik açısal konum girişine karşılık robot kolun uç noktasının konumunu verirken, ters kinematik uç noktasının istenen konumuna ait mafsallardaki açısal konumları vermektedir. İki serbestlik dereceli robot kolun düz kinematik denklemleri geometri yardımı ile kolayca bulunabilmektedir. Robotun uç 
noktasının $x$ ve $y$ koordinatlarına ait düz kinematik denklemleri Eşitlik (8) ve (9)'da sırasıyla verilmiştir.

$x=l_{1} \cos \theta_{1}+l_{2} \cos \left(\theta_{1}+\theta_{2}\right)$

$y=l_{1} \sin \theta_{1}+l_{2} \sin \left(\theta_{1}+\theta_{2}\right)$

Ters kinematik denklemlerinin edilmesi için bazı kurallar uygulanması gerekmektedir. Denavit Hartenberg tablosu oluşturulur ve dönüşüm matrisleri elde edilir. DH tablosunda bulunan parametreler mafsal tiplerine, sayısına ve sabit parametrelere göre farklılıklar gösterebilir. İki serbestlik dereceli robot kol için ters kinematik kuralları uygulanarak mafsal açısal konumları olan $\theta_{1}$ ve $\theta_{2}$ denklemleri elde edilmiştir, bu denklemkler Eşitlik (10) ve (11)'de sırasıyla verilmiştir [22].

$\theta_{1}=\operatorname{atan}\left(\frac{y}{x}\right) \pm \operatorname{atan}\left(\frac{l_{2} \sin \theta_{2}}{l_{1}+l_{2} \cos \theta_{2}}\right)$

$\theta_{2}= \pm \operatorname{acos}\left(\frac{x^{2}+y^{2}-l_{1}{ }^{2}-l_{2}{ }^{2}}{2 l_{1} l_{2}}\right)$

\subsection{PID Kontrol}

PID kontrolcüler uzun yıllardır endüstri de yaygın bir şekilde kullanılmaktadır. PID kontrolcülerde oransal, integral ve türevsel olmak üzere üç adet kazanç parametresi bulunur. Referans ve gerçek çıkış arasındaki hataya bağlı olarak kontrol sinyali üretilerek sisteme gönderilir. PID kontrol parametreleri geleneksel yöntemlerle belirlenebildiği gibi bu çalışmada olduğu gibi farklı yöntemlerle de belirlenebilmektedir. PID Kontolcü genel transfer fonksiyonu Eşitlik 11'de görülmektedir. $\mathrm{Bu}$ eşitlik düzenlendiği zaman $T_{i}$ ve $T_{d}$ katsayılarının oransal kazanç parametresi olan $K_{p}$ ile olan bağlantıları Eşitlik 12'deki integral kazanç $K_{i}$ ve türevsel kazanç $K_{d}$ parametrelerini verir [23].

$G(s)=K_{p}\left(1+\frac{1}{T_{i} s}+T_{d} s\right)$

$G(s)=K_{p}+\frac{K_{i}}{s}+K_{d} s$

İki serbestlik dereceli robot kolun açısal konumlarının kontrolü için iki adet PID kontrolcü tasarlanmıştır. Tasarlanan kontrol sistemi blok şeması Şekil 2'de verilmiştir. PID kontrol kazanç parametreleri ilk olarak deneme yanılma yöntemi ile belirlenmiştir, daha sonra GA ve AA kullanılarak belirlenmiştir ve sonuçlar karşılaştırılmıştır. 


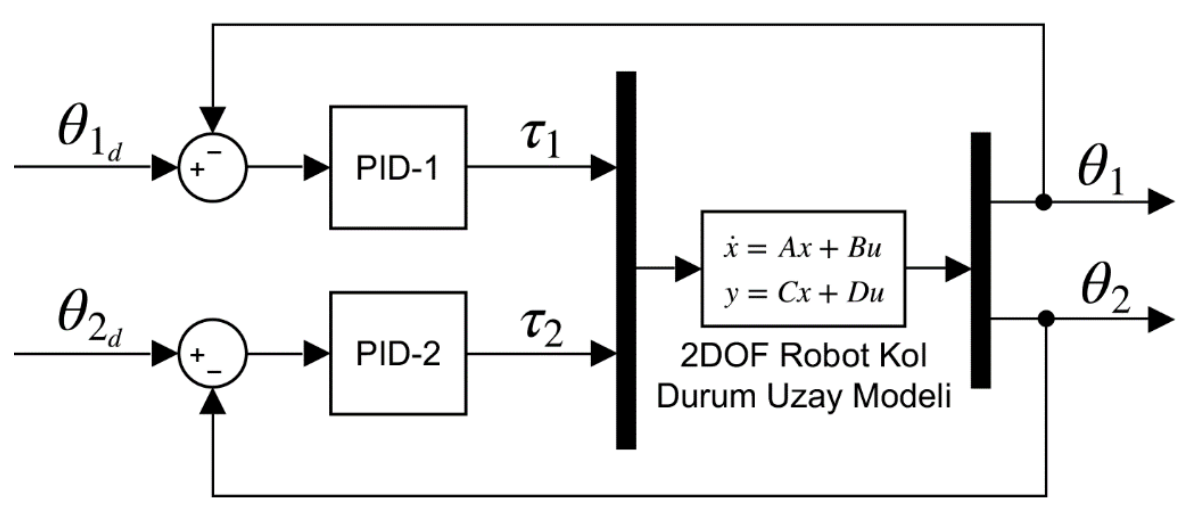

Şekil 2. PID Kontrol blok şeması.

\section{Arı Algoritması}

Karaboğa ve arkadaşları tarafindan öne sürülen Yapay Arı Algoritması (ABC) ile benzer mantığa sahip olan, Arı Algoritması (AA) D. T. Pham ve arkadaşları tarafından ilk olarak 2006 yılında önerilmiştir, arıların nektar ve su gibi kaynakları arama davranışlarını sürü zekâsı kullanarak öğrenme, hatırlama ve bilgi paylaşma özelliklerine benzetilerek sezgisel bir bakış açısı yakalanmıştır [24-28]. Elastik uzuvlu bir robot kolun kontrolü için bulanık mantık ve PID kontrolcüleri Arı Algoritması ile tasarlayan Pham ve Kalyoncu bu alandaki çalışmaların temellerini atmışlardır [29]. Kalyoncu ve arkadaşları Arı Algoritması ile çalışmaları sürdürülerek, konum ve denge kontrolünde geleneksel yöntemlere nazaran Arı Algoritması ile parametreleri belirlenen kontrolcülerin daha iyi sonuçlar verdiği gözlemlenmiştir [30-34].

Ar1 Algoritması birçok parametre içermektedir. Bu parametreler: kâşif arı sayısı (n), ziyaret edilen $n$ nokta içinden seçilen en uygun bölge sayısı $(m)$, seçilen $m$ bölge içindeki en iyi bölge sayısı (e), en iyi e bölgeye gönderilen arı sayısı (nep), kalan (m-e) bölgeye gönderilen arı sayısı ( $n s p)$, bölge boyutu ( $n g h)$ ve durdurma kriteri/iterasyon (itr) sayısidır.

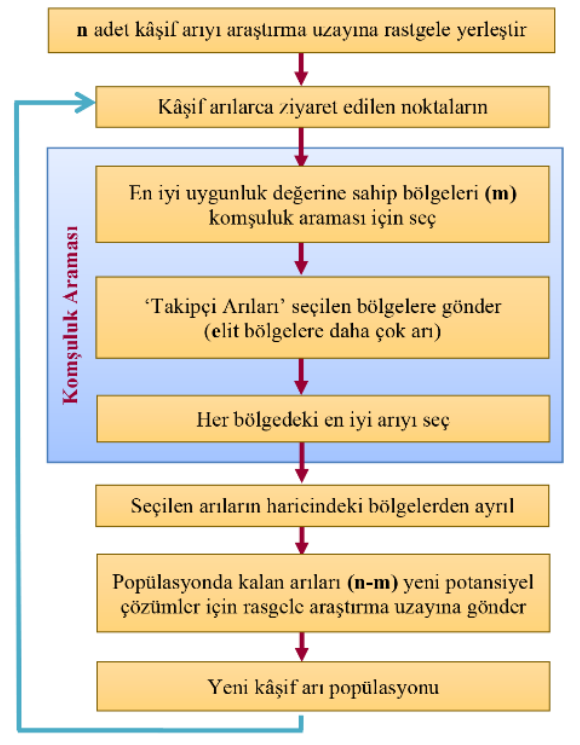

Şekil 3. Arı Algoritması akışs şeması 
Şekil 3'de Arı Algoritması akış şeması gösterilmektedir. Şekilde görüldüğü üzere bu döngü optimizasyonun durdurma kriteri (itr) sağlanana kadar devam eder. Bu çalışmada yer alan Arı Algoritması parametreleri Tablo 2'de gösterilmiştir.

Tablo 2. Arı Algoritması parametreleri

\begin{tabular}{|c|c|c|c|c|c|c|}
\hline itr & $n$ & $m$ & $e$ & nep & nsp & ngh \\
\hline 20 & 20 & 8 & 5 & 20 & 10 & 0.01 \\
\hline
\end{tabular}

Amaç fonksiyonu belirlenirken her iki konum çıkışının zaman cevapları, yükselme zamanı, tepe zamanı, maksimum aşma, maksimum değer ve kalıcı durum hataları göz önüne alınmış ve detaylı bir amaç fonksiyonu belirlenmiştir, Eşitlik (13)'de verilmiştir.

$J=\left(t r+t p+t s+M p+\theta_{\text {max }}+e s s\right)_{\theta_{1}}+\left(t r+t p+t s+M p+\theta_{\text {max }}+e s s\right)_{\theta_{2}}$

Ar1 Algoritmasını parametrelerinin ve PID parametrelerinin Tablo 3'de yer alan optimizasyon aralıklarının belirlenmesinde, sistemin zaman cevaplarının yanı sira ve PID parametrelerinin geleneksel yöntemlerle elde edilmesinden yararlanılmıştır.

Tablo 3. PID Kontrol parametrelerinin optimizasyon aralıkları

\begin{tabular}{|c|c|c|c|c|c|c|}
\hline & \multicolumn{3}{|c|}{ PID-1 } & \multicolumn{3}{c|}{ PID-2 } \\
\cline { 2 - 7 } & $K_{p}$ & $K_{i}$ & $K_{d}$ & $K_{p}$ & $K_{i}$ & $K_{d}$ \\
\hline Minimum & 0 & 0 & 0 & 0 & 0 & 0 \\
\hline Maksimum & 1000 & 1000 & 100 & 2000 & 2000 & 200 \\
\hline
\end{tabular}

\section{Sonuçlar}

Bu çalışmada, iki serbestlik dereceli bir robot kolun konum kontrolü için PID parametreleri Genetik Algoritma (GA) ve Arı Algoritması (AA) ile minimum konum hatası amaçlanarak belirlenmiştir. Deneme-yanılma, Genetik Algoritma ve Arı Algoritması ile belirlenen parametreler Tablo 4'de verilmiştir.

Tablo 4. Deneme-yanılma, GA ve AA ile belirlenen PID Parametreleri

\begin{tabular}{|c|c|c|c|c|}
\hline \multicolumn{2}{|c|}{ Kontrolcü } & Deneme-yanılma & Genetik Algoritma & Ari Algoritması \\
\hline \multirow{3}{*}{ PID-1 } & $K_{p}$ & 200 & 344.552 & 191.386 \\
\cline { 2 - 5 } & $K_{i}$ & 200 & 240.359 & 583.746 \\
\cline { 2 - 5 } & $K_{d}$ & 30 & 41.547 & 58.634 \\
\hline \multirow{3}{*}{ PID-2 } & $K_{p}$ & 800 & 1091.166 & 710.208 \\
\cline { 2 - 5 } & $K_{i}$ & 1000 & 1423.688 & 1576.271 \\
\cline { 2 - 5 } & $K_{d}$ & 100 & 150.598 & 168.405 \\
\hline
\end{tabular}

Deneme yanılma ve optimizasyon çalışmaları sonucunda belirlenen parametreler kullanılarak elde edilen sonuçlar, uzuvların açısal konumları $\theta_{1}$ ve $\theta_{2}$ 'nin konum-zaman grafikleri karşılaştırmalı olarak sırasıyla Şekil 4 ve Şekil 5'de verilmiştir. 


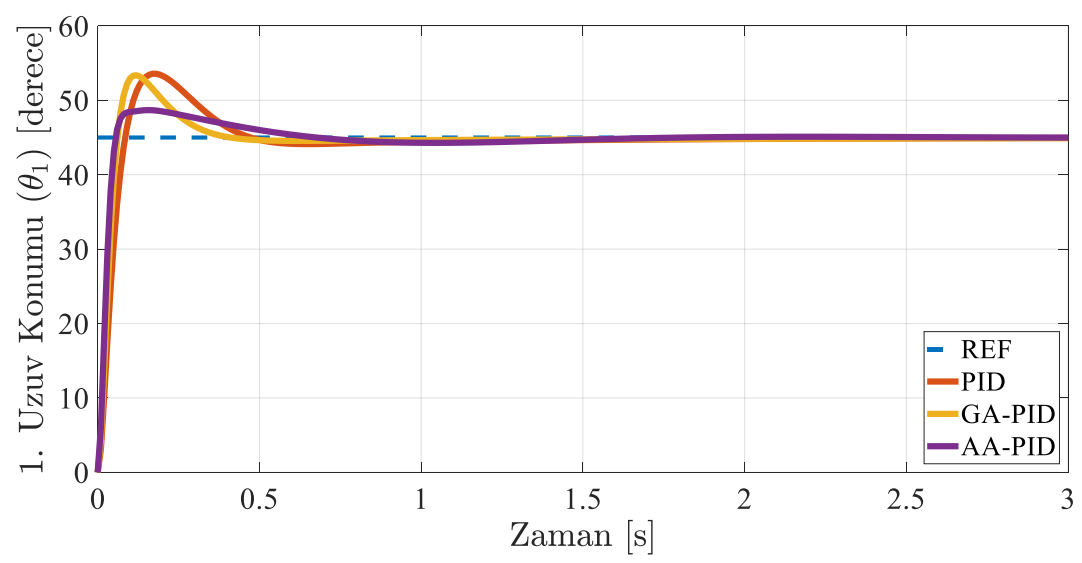

Şekil 4. 1. uzuv açısal konumu PID, GA-PID ve AA-PID Karşılaştırması.

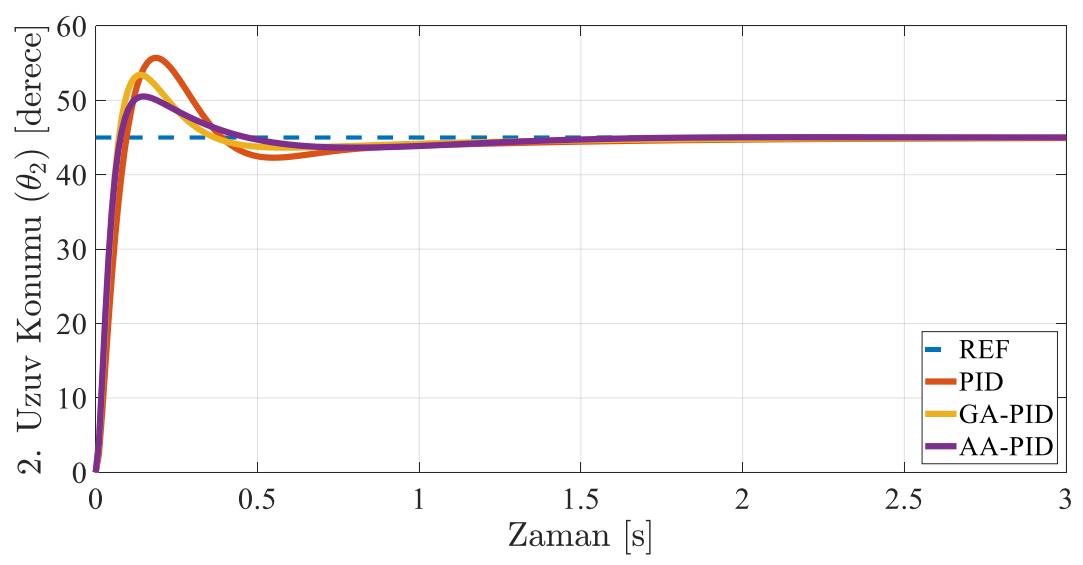

Şekil 5. 2. uzuv açısal konumu PID, GA-PID ve AA-PID Karşılaştırması.

PID parametreleri belirlendikten sonra kontrol şemasına robot kol sisteminin ters ve düz kinematikleri eklenmiştir ve robot kolun uç noktasının $x_{d}=1 \mathrm{~m}$ ve $y_{d}=1.5 \mathrm{~m}$ referans koordinatlarına gitmesi için kontrol çalışmaları yapılmıştır. Ters ve düz kinematikler eklenmiş olan kontrol sistemi şeması Şekil 6'da görülmektedir.

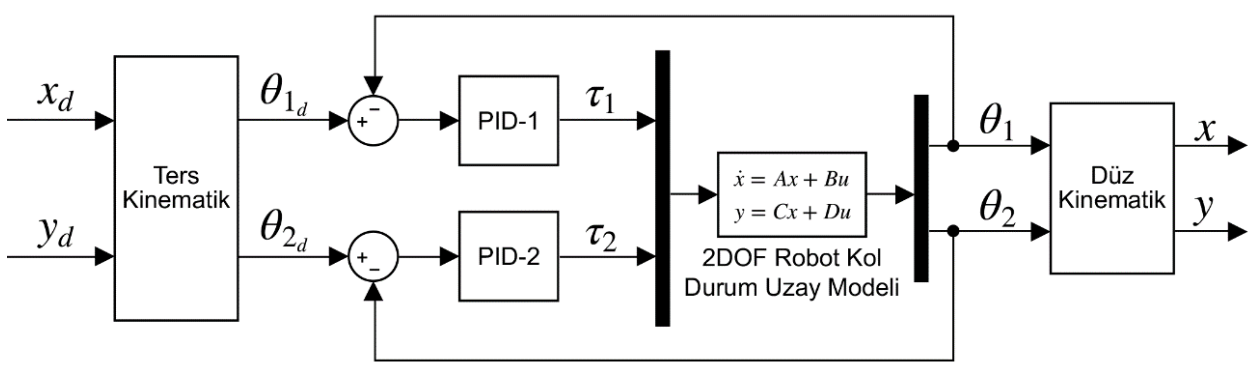

Şekil 6. PID Kontrol blok şeması ve ters-düz kinematik modeli.

Elde edilen sonuçlar, sistem performansının ölçülebilmesi için MATLAB/Simulink ortamında oluşturulan sistem modeli ile simüle edilerek yükselme zamanı, yerleşme zamanı, tepe zamanı, maksimum aşma ve kalıcı durum hatası gibi performans değerleri elde edilmiş ve Tablo 5'de sunulmuştur. 
Tablo 5. PID, GA-PID ve BA-PID Kontrolcü performans değerleri

\begin{tabular}{|c|c|c|c|c|}
\hline Değişken & Zaman Cevabı & PID & GA-PID & AA-PID \\
\hline \multirow{5}{*}{$\theta_{1}$} & Yükselme Zamanı $\left(\mathrm{t}_{\mathrm{r}}\right)$ [sn] & 0.0677 & 0.0476 & 0.0423 \\
\hline & Yerleşme Zamanı $\left(\mathrm{t}_{\mathrm{s}}\right)[\mathrm{sn}]$ & 0.9195 & 0.3393 & 0.5114 \\
\hline & Tepe Zamanı $\left(t_{p}\right)[s n]$ & 0.196 & 0.1330 & 0.162 \\
\hline & Maksimum Aşma [\%] & 21.3518 & 36.9134 & 9.4855 \\
\hline & Kalıcı Durum Hatası $\left(\mathrm{e}_{\mathrm{ss}}\right)$ [der] & 0 & 0 & 0 \\
\hline \multirow{5}{*}{$\theta_{2}$} & Yükselme Zamanı $\left(\mathrm{t}_{\mathrm{r}}\right)$ [sn] & 0.0688 & 0.0507 & 0.0524 \\
\hline & Yerleşme Zamanı $\left(\mathrm{t}_{\mathrm{s}}\right)[\mathrm{sn}]$ & 1.1466 & 0.9376 & 1.1324 \\
\hline & Tepe Zamanı $\left(t_{p}\right)[s n]$ & 0.188 & 0.1400 & 0.149 \\
\hline & Maksimum Aşma [\%] & 24.3587 & 61.1747 & 12.5834 \\
\hline & Kalıcı Durum Hatası $\left(\mathrm{e}_{\mathrm{ss}}\right)$ [der] & 0 & 0 & 0 \\
\hline \multirow{5}{*}{$x$} & Yükselme Zamanı $\left(\mathrm{t}_{\mathrm{r}}\right)$ [sn] & 0.0596 & 0.0423 & 0.044 \\
\hline & Yerleşme Zamanı $\left(\mathrm{t}_{\mathrm{s}}\right)[\mathrm{sn}]$ & 1.4815 & 1.1058 & 1.3321 \\
\hline & Tepe Zamanı $\left(t_{p}\right)[s n]$ & 0.192 & 0.1360 & 0.153 \\
\hline & Maksimum Aşma [\%] & 39.4093 & 34.4476 & 18.9473 \\
\hline & Kalıc1 Durum Hatas1 $\left(\mathrm{e}_{\mathrm{ss}}\right)[\mathrm{m}]$ & 0 & 0 & 0 \\
\hline \multirow{5}{*}{$y$} & Yükselme Zamanı $\left(\mathrm{t}_{\mathrm{r}}\right)[\mathrm{sn}]$ & 0.0605 & 0.0436 & 0.0396 \\
\hline & Yerleşme Zamanı $\left(\mathrm{t}_{\mathrm{s}}\right)$ [sn] & 0.3774 & 0.2905 & 0.3604 \\
\hline & Tepe Zamanı $\left(t_{p}\right)[s n]$ & 0.213 & 0.1290 & 0.164 \\
\hline & Maksimum Aşma [\%] & 5.785 & 6.1694 & 3.5034 \\
\hline & Kalıcı Durum Hatası $\left(\mathrm{e}_{\mathrm{ss}}\right)[\mathrm{m}]$ & 0 & 0 & 0 \\
\hline
\end{tabular}

Robot kolun istenen konuma ulaşabilmesi için geren uzuv açıları ters kinematik ile hesaplanarak kontrol sistemine giriş olarak verilmiştir. Ters kinematik ile elde edilen açısal konumların karşılaştırmalı sonuçları Şekil 7 ve Şekil 8'de verilmiştir.

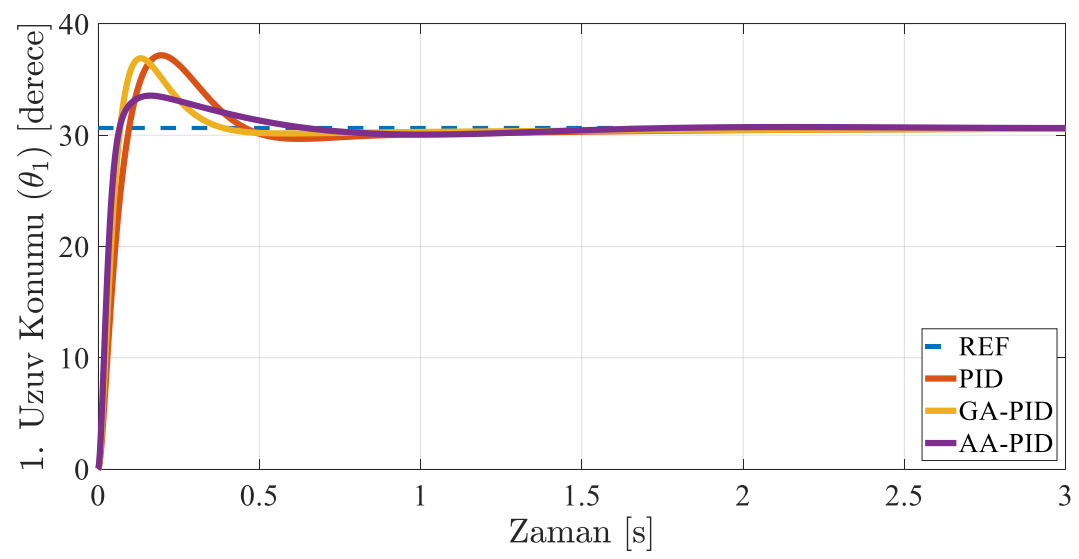

Şekil 7. 1. uzuv açısal konumu PID, GA-PID ve AA-PID Karşılaştırması. 


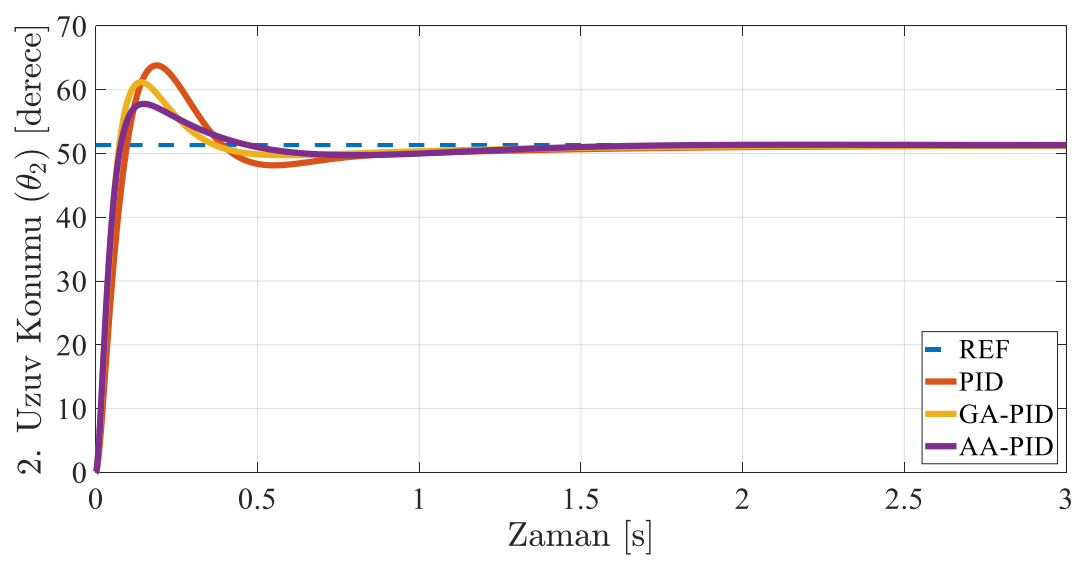

Şekil 8. 2. uzuv açısal konumu PID, GA-PID ve AA-PID Karşılaştırması.

Kontrol çalışmaları sonucunda elde edilen uzuvların açısal konumları, düz kinematik modeli kullanılarak robotun ulaştığı noktanın koordinatları elde edilmiş ve referans değerler ile karşılaştırılmıştır. Robot uç noktasının $x$ ve $y$ koordinatlarına ait sonuçlar sırasıyla Şekil 9 ve Şekil 10'de verilmiştir.

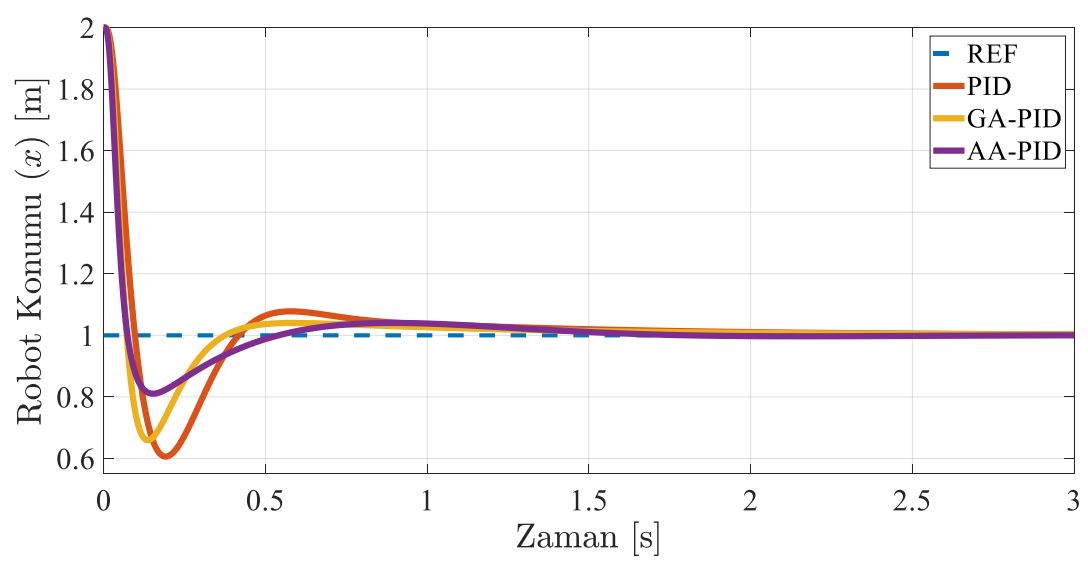

Şekil 9. Robot ucu $x$ konumu PID, GA-PID ve AA-PID Karşılaştırması.

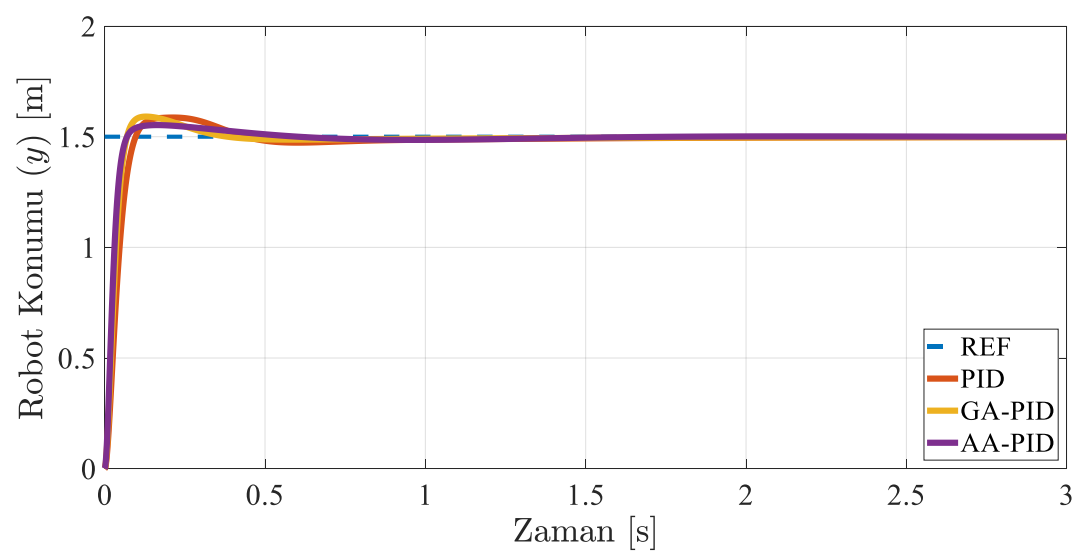

Şekil 10. Robot ucu y konumu PID, GA-PID ve AA-PID Karşılaştırması.

Robot kolun istenilen konuma gelmesi için gerekli tork değerleri, Genetik Algoritma ve Arı Algoritması ile belirlenen PID parametreleri kullanılarak sistemin simüle edilmesi 
sonucu elde edilmiş ve 1. ve 2. uzva ait tork değerleri sırası ile Şekil 11 ve Şekil 12'de verilmiştir.

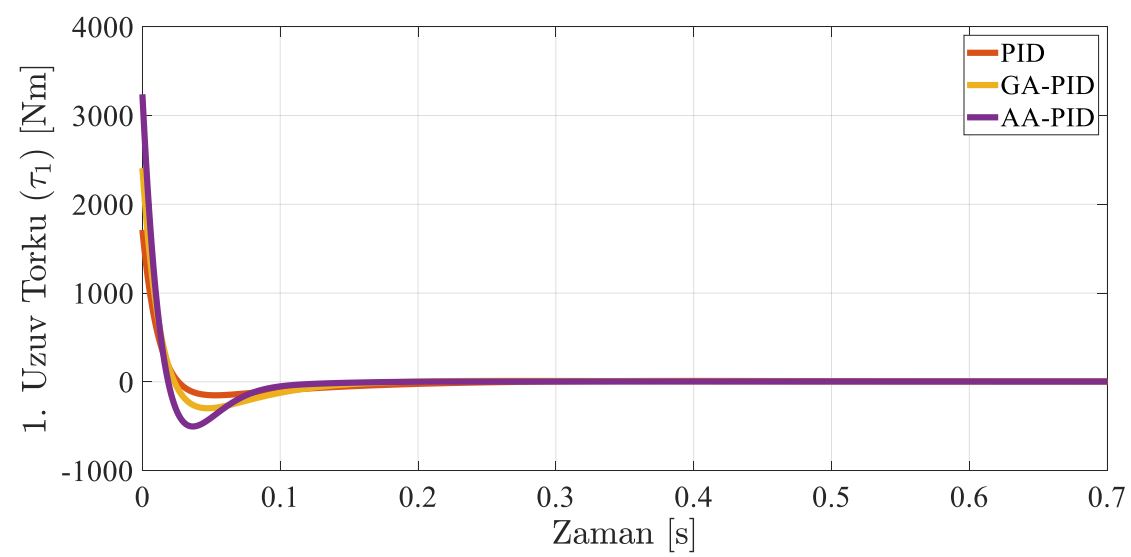

Şekil 11. Robot ucu y konumu PID, GA-PID ve AA-PID Karşılaştırması.

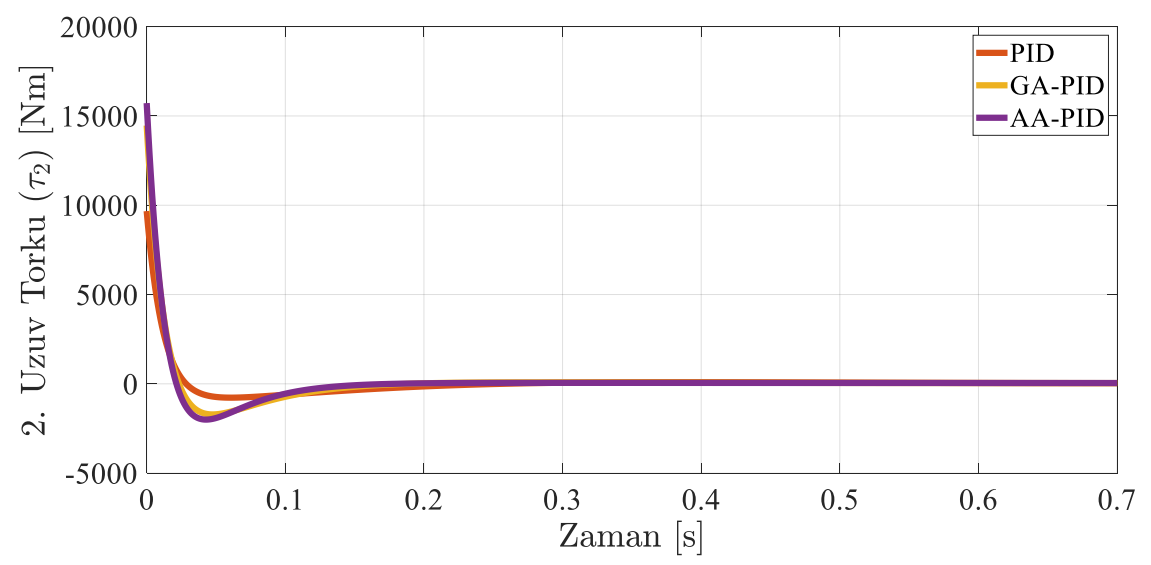

Şekil 12. Robot ucu y konumu PID, GA-PID ve AA-PID Karş1laştırması.

Genetik Algoritma ve Arı Algoritması kullanılarak yapılan optimizasyonlara ait amaç fonksiyonu değişimi Şekil 13'de verilmiştir.

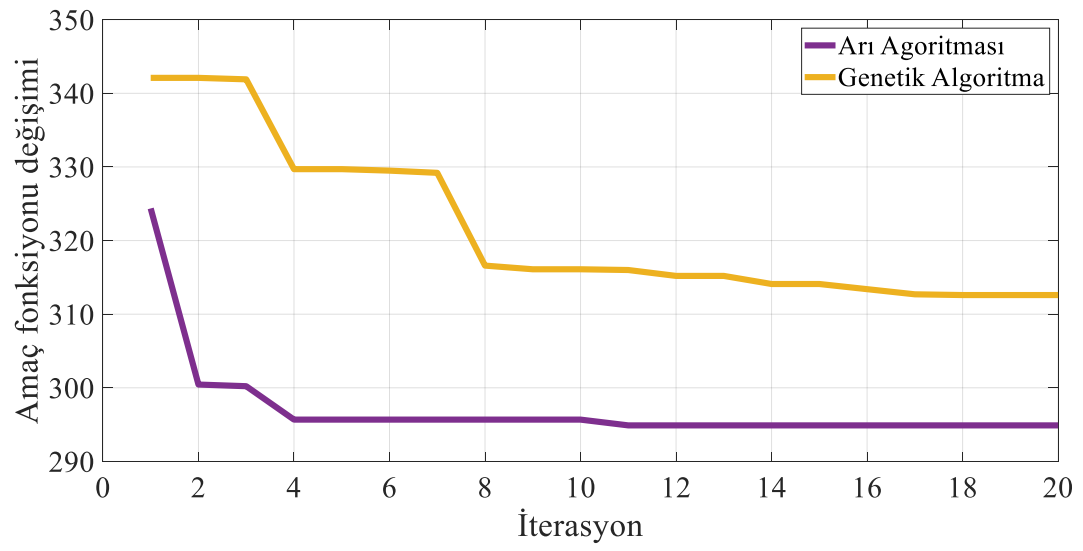

Şekil 13. GA ve AA Amaç fonksiyonu değiş̧imi. 


\section{Tartışma}

Bu çalışmada iki serbestlik dereceli bir robot kolun konum kontrolü yapılmıştır. Konum kontrolünün yapılabilmesi için PID kontrolcü tasarlanmıştır ve kontrol parametreleri ilk olarak deneme yanılma yoluyla elde edilmiştir. Daha sonra PID parametrelerinin belirlenmesinde Genetik Algoritma (GA) ve Arı Algoritması (AA) kullanılmıştır. Matlab/Simulink ortamında yapılan benzetim çalışmaları sonucunda her iki algoritmanın da PID kontrol parametrelerinin belirlenmesinde başarılı olduğu ve robot kolun istenilen referans konumlara gelebildiği gözlemlenmiştir. Bununla beraber, Arı Algoritması ile belirlenen parametreler ile yapılan simülasyon çalışmalarında daha az maksimum aşmalar elde edildiği gözlenmiştir. Arı Algoritması (AA) parametrelerinin değiştirilmesi ile farklı konfigürasyonlar elde edilerek, farklı amaç fonksiyonun belirlenerek, farklı kontrolcülerin de optimize edilebileceği, deneysel ve benzetim çalışmalarında başarılı sonuçlar verebileceği öngörülmektedir.

\section{Kaynaklar}

[1] Berg, A., Buffie, E. F., and Zanna, L.-F., Robots, growth, and inequality, Finance \& Development, 53, 3, 10-13, (2016).

[2] Enescu, M. L. and Alexandru, C., Modeling and simulation of a 6 DOF robot, Advanced Materials Research, 463, 1116-1119, (2012).

[3] Kim, H.-S. and Song, J.-B., Low-cost robot arm with 3-DOF counterbalance mechanism, 2013 IEEE International Conference on Robotics and Automation, 4183-4188, Karlsruhe, (2013).

[4] S. Klug, O. von Stryk and B. Mohl, Design and Control Mechanisms for a 3 DOF Bionic Manipulator, The First IEEE/RAS-EMBS International Conference on Biomedical Robotics and Biomechatronics, BioRob 2006., 450-454, Pisa, (2006).

[5] Wu, J., Wang, J., Li, T., and Wang, L., Analysis and application of a 2-DOF planar parallel mechanism, Journal of Mechanical Design, 129, 4, 434-437, (2007).

[6] Amer, A. F., Sallam, E. A., and Elawady, W. M., Adaptive fuzzy sliding mode control using supervisory fuzzy control for 3 DOF planar robot manipulators, Applied Soft Computing, 11, 8, 4943-4953, (2011).

[7] Çakan, A. and Botsali, F. M., Inverse kinematics analysis of a puma robot by using MSC Adams, VI International Conference Industrial Engineering and Environmental Protection, 274-277, Zrenjanin, (2016).

[8] Spong, M. W., Hutchinson, S., and Vidyasagar, M., Robot modeling and control, John Wiley \& Sons, (2006).

[9] Cervantes, I. and Alvarez-Ramirez, J., On the PID tracking control of robot manipulators, Systems \& Control Letters, 42, 1, 37-46, (2001).

[10] İlgen, S., Durdu, A., Gülbahçe, E., and Çakan, A., İki Linkli Robot Manipülatörün Modellenmesi ve Bozucu Etki Altında Yörünge Kontrolü, 4th International Symposium on Multidisciplinary Studies and Innovative Technologies (ISMSIT), 1-5, Istanbul, (2020).

[11] ÖNEN, Ü., Cakan, A., and Ilhan, I., Performance comparison of optimization algorithms in LQR controller design for a nonlinear system, Turkish Journal of Electrical Engineering \& Computer Sciences, 27, 3, 1938-1953, (2019). 
[12] KILIÇ, F., BİCAKCI, S., and Güneş, H., Ters sarkacın uyarlamalı kazançlı bağımsız yüzeyli kayan kip yöntemi ile denetimi, Balıkesir Üniversitesi Fen Bilimleri Enstitüsü Dergisi, 21, 2, 610-622, (2019).

[13] Lochan, K. and Roy, B., Control of two-link 2-dof robot manipulator using fuzzy logic techniques: A review, 4th International Conference on Soft Computing for Problem Solving, 335, 499-511, New Delhi, (2015).

[14] Ogata, K., Modern control engineering. Prentice hall, (2010).

[15] Çalgan, H., Yaman, R., İlten, E., and Demirtaş, M., Fırçasız DA motorunun hız kontrolünde PI katsayılarının Pareto tabanlı çok amaçlı optimizasyonu, Balıkesir Üniversitesi Fen Bilimleri Enstitüsü Dergisi, 20, 2, 330-346, (2018).

[16] Civelek, Z., Optimization of fuzzy logic (Takagi-Sugeno) blade pitch angle controller in wind turbines by genetic algorithm, Engineering Science and Technology, an International Journal, 23, 1, 1-9, (2020).

[17] Liang, H., Zou, J., Zuo, K., and Khan, M. J., An improved genetic algorithm optimization fuzzy controller applied to the wellhead back pressure control system, Mechanical Systems and Signal Processing, 142, 106708, (2020).

[18] Solihin, M. I., Tack, L. F., and Kean, M. L., Tuning of PID controller using particle swarm optimization (PSO), International Journal on Advanced Science, Engineering and Information Technology, 1, 4, 458-461, (2011).

[19] Oshaba, A. S., Ali, E. S., and Abd Elazim, S. M., "PI controller design using ABC algorithm for MPPT of PV system supplying DC motor pump load, Neural Computing and Applications, 28, 2, 353-364, (2015).

[20] Karaboga, D. and Akay, B., "Proportional-integral-derivative controller design by using artificial bee colony, harmony search, and the bees algorithms, Proceedings of the Institution of Mechanical Engineers, Part I: Journal of Systems and Control Engineering, 224, 7, 869-883, (2010).

[21] Mustafa, A. M. and Al-Saif, A., Modeling, simulation and control of 2-R robot, Global Journal of Research In Engineering, 14, 1H, (2014).

[22] Kucuk, S. and Bingul, Z., Robot kinematics: Forward and inverse kinematics. INTECH Open Access Publisher, (2006).

[23] Ang, K. H., Chong, G., and Li, Y., PID control system analysis, design, and technology, IEEE Transactions on Control Systems Technology, 13, 4, 559576, (2005).

[24] Pham, D.T., Koç, E., Ghanbarzadeh, A., Otri, S., Rahim,S., Zaidi, M., The Bees Algorithm A Novel Tool for Complex Optimisation Problems, 2nd International Virtual Conference on Intelligent Production Machines and Systems, 454-461, (2006).

[25] Karaboga, D., An idea based on honey bee swarm for numerical optimization, Citeseer, (2005).

[26] Karaboga, D. and Akay, B., "A comparative study of artificial bee colony algorithm," Applied Mathematics and Computation, 214, 1, 108-132, (2009).

[27] Karaboga, D. and Basturk, B., A powerful and efficient algorithm for numerical function optimization: artificial bee colony $(\mathrm{ABC})$ algorithm, Journal of Global Optimization, 39, 3, 459-471, (2007).

[28] Karaboga, D. and Basturk, B., On the performance of artificial bee colony (ABC) algorithm, Applied soft computing, 8, 1, 687-697, (2008).

[29] Pham, D.T., Koç, E., Kalyoncu, M., Tınkır, M., Hierarchical PID Controller Design for a Flexible Link Robot Manipulator Using the Bees Algorithm, 6th International Symposium on Intelligent Manufacturing Systems, 757-765, Sakarya, (2008). 
[30] Pham, D. and Kalyoncu, M., Optimisation of a fuzzy logic controller for a flexible single-link robot arm using the Bees Algorithm, 7th IEEE International Conference on Industrial Informatics, 475-480, (2009).

[31] Bakırcıoğlu, V., Şen, M., and Kalyoncu, M., Dört Ayaklı Robotun Bir Bacağ1 İçin PID Kontrolcü Tasarımı ve Arı Algoritması Kullanarak Optimizasyonu, Uluslararası Katılımlı 17. Makina Teorisi Sempozyumu, İzmir, (2015).

[32] Arif Şen, M., Tinkir, M., and Kalyoncu, M., Optimisation of a PID controller for a two-floor structure under earthquake excitation based on the bees algorithm, Journal of Low Frequency Noise, Vibration and Active Control, 37, 1, 107127, (2018).

[33] Şen, M. A., Bakırcioğlu, V., and Kalyoncu, M., Performances Comparison of The Bees Algorithm and Genetic Algorithm for PID Controller Tuning, 5th International Conference on Mechatronics and Control Engineering ICMCE '16, (2016).

[34] Sen, M. A. and Kalyoncu, M., Optimisation of a PID Controller for an Inverted Pendulum Using the Bees Algorithm, Applied Mechanics and Materials, 789790, 1039-1044, (2015). 